MRI-PHY/P970615

hep-ph/9707239

\title{
TESTING GAUGE-GRAVITINO COUPLING IN GAUGE-MEDIATED SUPERSYMMETRY BREAKING THROUGH SINGLE PHOTON EVENTS
}

\author{
Amitava Datta*円, Aseshkrishna Datta*ף, Anirban Kundu**\}, \\ Biswarup Mukhopadhyaya** ${ }^{*}$, and Sourov Roy** \\ * Physics Department, Jadavpur University, Calcutta - 700 032, India \\ ** Mehta Research Institute, Chhatnag Road, Jhusi, Allahabad - 221 506, India
}

\begin{abstract}
We show that the process $e^{+} e^{-} \longrightarrow \gamma+$ missing energy, arising from the pair production of neutralinos, can probe the $\gamma-\tilde{\gamma}-\tilde{G}$ as well as the $Z-\tilde{Z}-\tilde{G}$ couplings in Gauge Mediated Supersymmetry Breaking models. This enables one to study the mutual relationship of the Goldstino couplings of the different gauginos, a feature whose testability has not been emphasized so far. The Standard Model backgrounds get suppresed with the use of a right polarized electron beam. The energy and angular distribution of the emitted photon can distinguish such models from the minimal supersymmetric theory and its variants.
\end{abstract}

\footnotetext{
${ }^{1}$ Electronic address: adatta@juphys.ernet.in

${ }^{2}$ Electronic address: asesh@juphys.ernet.in

${ }^{3}$ Electronic address: akundu@mri.ernet.in

${ }^{4}$ Electronic address: biswarup@mri.ernet.in

${ }^{5}$ Electronic address: sourov@mri.ernet.in
} 
Distinguishing between various types of supersymmetry (SUSY) breaking schemes is one crucial task ahead of those who are engaged in the search for SUSY in nature [1]. Models with Gauge Mediated Supersymmetry Breaking (GMSB) [2] have recently attracted considerable at-

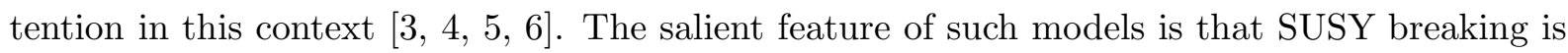
communicated radiatively to the observable sector via ordinary gauge interactions, as opposed to more conventional theories where gravitation plays a decisive role. This is usually implemented by introducing a messenger sector, consisting of vectorlike quark and lepton superfields $\hat{\psi}$. The messenger superpotential contains a term of the form of $\lambda S \hat{\bar{\psi}} \hat{\psi}$, where $S$ is a gauge singlet superfield. The scalar and auxiliary components of $S$ acquire vacuum expectation values (VEV) via interactions with the hidden sector. This lifts the mass degeneracy between the messenger fermions and sfermions. The observable gauginos and sfermions acquire their masses at the one- and two-loop levels respectively, with the messenger fields participating in the loops. The gaugino, squark and slepton masses are thus all related. An advantage of this scheme is that flavour-diagonal sfermion masses are induced at a rather low energy scale $(\sim 100 \mathrm{TeV}$ or so). This suppresses flavour-changing neutral currents which could be potentially dangerous in supergravity type models due to interactions of heavy fields above the Grand Unification scale [7]. Here the gravitino $(\tilde{G})$ becomes very light $(\sim 1 \mathrm{eV})$. Therefore, the lightest Standard Model superparticle can decay into it. Thus the so-called lightest SUSY particle (LSP) of the conventional model now becomes the next lightest SUSY particle (NLSP), and is unstable; in fact, in the minimal version of GMSB, it can decay within the detector over a large part of the parameter space $\rrbracket$, making the observable signals radically different from those of the minimal

\footnotetext{
${ }^{1}$ As can be seen, for example, from Bagger et al. in ref [f], a neutralino NLSP $\chi$ can decay within the detector so long as the parameter $M / \Lambda$ (defined later in the text) is not appreciably higher than 100 , and if $\left|\vec{p}_{\chi}\right| \sim m_{\chi}$. The decay length can possibly be larger if the gravitino decay constant gets contributions from additional fields present in the SUSY breaking sector, as discussed by Ambrosanio et al. in ref. [9].
} 
SUSY Standard Model (MSSM).

If there is one generation of messengers, the lightest neutralino is the NLSP. With a larger number of messenger generations, a right slepton may take that place. Here we are considering the case with a neutralino NLSP, where the messenger fields form one complete $5+\overline{5}$ of $S U(5)$. The entire mass spectrum as well as the chargino and neutralino mixing matrices are determined by the messenger mass scale $M$, and more crucially, by a parameter $\Lambda$ (the ratio of the VEVs of the scalar and auxiliary components of $S$ ), together with the Higgsino mass parameter $\mu, \tan \beta$ (the ratio of the two Higgs' VEVs) and the number of messenger generations.

If a neutralino is the NLSP, it is almost always dominated by the Bino component. The main decay mode of such an NLSP is $\chi_{1}^{0} \longrightarrow \gamma \tilde{G}$. Based on this, signals of GMSB have been predicted for colliders like the Fermilab Tevatron, LEP-2 and the Next Linear Collider (NLC) [8, 9, 10]. However, the possibility of testing the components of $\chi_{1}^{0}$ other than the photino has so far been only briefly commented upon (for example, by Dimopoulos et al. in reference [5] and Ambrosanio et al. in reference [8]). The Zino component of $\chi_{1}^{0}$ will give rise to the decay $\chi_{1}^{0} \longrightarrow Z \tilde{G}$ if $m_{\chi_{1}^{0}}>m_{Z}$, although the branching ratio will be rather small. If the signature of such a decay is available, then one can address two important issues related to the structure of GMSB. First, it will probe the composition of the NLSP. Secondly, it will provide a test for the coupling of the $Z-\tilde{Z}$ current with the longitudinal component of $\tilde{G}$, whose strength is related to similar coupling of the $\gamma-\tilde{\gamma}$ current [1]. In this paper we suggest a new way of performing both these tests by considering the process $e^{+} e^{-} \longrightarrow \chi_{1}^{0} \chi_{1}^{0} \longrightarrow \gamma \tilde{G} Z \tilde{G}$, followed by the $Z$ decaying invisibly into a pair of neutrinos. In this way, a small but finite Zino component of the NLSP shows up in single photon events (SPE) in high-energy $e^{+} e^{-}$colliders, the NLC in particular. As we shall demonstrate here, such events are easier to isolate from the backgrounds if polarized 
electron beams are used.

The advantage of this channel is that SPEs are in any case observed with great care in $e^{+} e^{-}$colliders for testing QED as well as physics beyond the Standard Models with heavy stable neutral particles. In the context of GMSB, the signal proposed by us will also serve as a confirmatory test while more copious two-photon events are likely to be the primary signal.

The production cross-section of $\chi_{1}^{0}$ pairs at high energy $e^{+} e^{-}$colliders as a function of the centre-of-mass energy $\sqrt{s}$ is presented in Figure 1 for both polarized and unpolarized electron beams. Here the controlling factors are the $\chi_{1}^{0}$ mass $\left(m_{\chi_{1}^{0}}\right)$ and the neutralino mixing angles. These are essentially determined by $\Lambda$ throughout the parameter space of our interest. This is because in this region $\chi_{1}^{0}$ is dominantly Bino and hence the influence of $\mu$ or $\tan \beta$ is small. The only exception occurs if $|\mu|$ is small compared to the gaugino mass parameters, in which case $\chi_{1}^{0}$ has a substantial Higgsino part. Then the $t$-channel slepton exchange cross section is suppressed by the electron mass and the signal becomes very weak. In all the numerical results presented here, we have used $\mu=-300 \mathrm{GeV}, \tan \beta=10$ and $M / \Lambda=2$.

As long as the gravitino mass is negligible compared to the relevant energy scale, it can be approximated by its Goldstino components. Since the Goldstino coupling to a particle-sparticle pair is universal in form, $Z-\tilde{Z}-\tilde{G}$ and $\gamma-\tilde{\gamma}-\tilde{G}$ couplings are related [12]. $B\left(\chi_{1}^{0} \longrightarrow \gamma \tilde{G}\right)$ and $B\left(\chi_{1}^{0} \longrightarrow Z \tilde{G}\right)$ are thus computed in a straightforward way. Some representative branching ratios are listed in Table 1. It can be seen from the table that over a large region of the parameter space $B\left(\chi_{1}^{0} \longrightarrow Z \tilde{G}\right)$ is small but non-negligible. For relatively large $m_{\chi_{1}^{0}}$, it can be as large as $23 \%$. However, in these cases the production cross-section is suppressed due to a correspondingly large selectron mass which occurs in the $t$ and $u$-channel propagators.

¿From Figure 1 and Table 1 , it is clear that at $\sqrt{s}=500 \mathrm{GeV}$, the signal cross section 
without any kinematical cut is of the order of a few $\mathrm{fb}$. The main irreducible Standard Model background comes from $e^{+} e^{-} \longrightarrow \nu \bar{\nu} \gamma$ whose cross section is $5.7 \mathrm{pb}$ for unpolarized beams [13]. This is large enough to swamp the signals.

The situation improves somewhat with the introduction of kinematical cuts. Following [14] we have introduced a set of cuts as given below:

$$
95 \mathrm{GeV}<E_{\gamma}<225 \mathrm{GeV}, 40^{\circ}<\theta_{\gamma}<140^{\circ} .
$$

These cuts also take care of the background from the radiative Bhabha scattering where the charged particles in the final states are lost in the beam pipe [15]. The signal cross section with these cuts are plotted in Figure 2. Only for a limited region of the parameter space the statistical significance $S / \sqrt{B}$ rises above 2 , where $S$ and $B$ are respectively the number of signal and background events corresponding to an integrated luminosity of $30 \mathrm{fb}^{-1}$. By strengthening the lower cut on $E_{\gamma}$ the significance increases somewhat but is always well below 5 . The situation does not improve by using alternative sets of cuts, e.g., the one proposed in [15]. One, therefore, is forced to the conclusion that the situation is not optimistic for unpolarized beams.

The signal improves drastically when polarized electron beams are available [16]. In this case, the irreducible Standard Model background from $\nu \bar{\nu} \gamma$ is heavily suppressed since the $t$ channel $W$-exchange diagrams do not contribute anymore. The signal, on the other hand, is jacked up due to the larger hypercharge of the right-handed gauge singlet electron coupling with the Bino-dominated NLSP. Using the above cuts, some representative signal cross sections with $100 \%$ right-polarized electrons at $\sqrt{s}=500 \mathrm{GeV}$ are plotted in Figure 3. Also plotted are the values corresponding to different significance levels. Here we have conservatively assumed an integrated luminosity of $10 \mathrm{fb}^{-1}$ with polarized beams. It is seen that for $130 \mathrm{GeV} \leq m_{\chi_{1}^{0}} \leq$ $210 \mathrm{GeV}$, the signal is above the $5 \sigma$ level. We have also checked that the other source of standard 
model backgrounds, namely, $e^{+} e^{-} \longrightarrow Z Z \gamma$ followed by both the Z's decaying invisibly, is not significant for the polarized signals even at a $10 \sigma$ level [17]. Figures 2 and 3 also confirm the fact that the dependence of the signal on $\mu$ is negligible for $|\mu|$ larger than the gaugino mass parameters. It should be emphasized that an even larger region of the parameter space can be explored if (a) a larger $\sqrt{s}$ is available, and (b) one has a larger integrated luminosity. Thus SPEs provide us with a strong handle on the $Z-\tilde{Z}-\tilde{G}$ interaction in the GMSB scenario.

However, the task of distinguishing GMSB from conventional SUSY scenarios is still to be accomplished. This is important because the latter may also lead to an excess of SPEs at the NLC. The relevant processes there are 14, 18.

$$
\begin{aligned}
\text { i) } e^{+} e^{-} & \longrightarrow \chi_{1}^{0} \chi_{1}^{0} \gamma \\
\text { ii) } e^{+} e^{-} & \longrightarrow \tilde{\nu} \tilde{\bar{\nu}} \gamma \\
\text { iii) } e^{+} e^{-} & \longrightarrow \chi_{i}^{0} \chi_{j}^{0} \gamma \quad(i, j=1,2 ; i=j \neq 1)
\end{aligned}
$$

Processes (ii) and (iii) occur in the special case where $\tilde{\nu}$ and $\chi_{2}^{0}$ decay invisibly (the "Virtual LSP" scenario [19]).

In Figure 4 we present the uncut cross sections with right polarized electron beams for the above processes. The relevant formulae can be obtained from the Appendix of [14] by taking limits appropriate for right polarized beams. We have chosen the SUSY parameters in such a way as to keep the MSSM contribution on the higher side so that the challenge of distinguishing GMSB signals from them becomes most obvious. These include three cases where the invisible final state comes from the lightest neutralino (LSP), the LSP and the $\tilde{\nu}$ and the LSP, the $\tilde{\nu}$ and the second lightest neutralino. The $\tilde{\nu}$ contributes when its dominant decay is invisible through the channel $\tilde{\nu} \longrightarrow \chi_{1}^{0} \nu$. In such a case, $\chi_{2}^{0}$ may also become invisible if its dominant decay mode is $\chi_{2}^{0} \longrightarrow \nu \tilde{\bar{\nu}}$ It is seen from Figure 4 that the size of this signal is larger than that of GMSB, 
and can very well mimic it. Thus, the size of the signal alone cannot be the deciding factor in discriminating between the two scenarios.

The two scenarios, however, can be distinguished from the energy and angular distributions of the final state photon. We present in Figure 5 the $E_{\gamma}$ distributions in GMSB as well as in MSSM, subject to the angular cut $40^{\circ}<\theta_{\gamma}<140^{\circ}$. The curve corresponding to GMSB is almost flat and shows the end-points characteristic of two-body decays. Moreover, it extends to higher values of $E_{\gamma}$ compared to the MSSM distribution. On the other hand, the MSSM curves with different choices of SUSY parameters show a continuous fall with energy, which can be clearly distinguished from the previous case.

In Figure 6, we show the angular distribution in the two scenarios subject to the energy cuts $95 \mathrm{GeV}<E_{\gamma}<225 \mathrm{GeV}$. The MSSM distributions are well-populated for photons relatively closer to the beam pipe with a distinct dip in the central region. On the contrary, the GMSB signal shows exactly the opposite trend. It may be argued that the $Z-\tilde{Z}-\tilde{G}$ may also be probed by processes where the $Z$ decays into a pair of jets. In such cases, the signal size will definitely be larger. The dominant background in this case will come from $e^{+} e^{-} \longrightarrow q \bar{q} \gamma$. It may be naively argued that a missing energy cut can discriminate the GMSB signal from the background. However, jet fragmentation, mismeasurement of jet momenta, soft gluon radiation etc. make the computation of the background much more cumbersome and uncertain.

We have repeated the calculation by varying the degree of polarization of the $e^{-}$beam. Our main conclusions remain qualitatively unaffected as long as more than $90 \%$ right-polarized electron beams are available.

In conclusion, we reiterate that an excess of single photon events at the NLC with polarized electron beams may provide a strong evidence for the $Z-\tilde{Z}-\tilde{G}$ coupling which is an inescapable 
consequence of the GMSB scenario. The Zino component of the NLSP can also be tested by the same signal. The signal is distinguishable not only from the Standard Model background but also from similar signals arising in MSSM without a light gravitino.

Amitava Datta's work is supported by the Department of Science and Technology, Government of India, under grant no. SP/S2/K-07/92. The work of Aseshkrishna Datta has been supported by the Council for Scientific and Industrial Research, India. AK and SR acknowledge the hospitality of the Theory Group, Saha Institute of Nuclear Physics, Calcutta, and BM and SR thank the Physics Department, Jadavpur University, where part of this work was done. 


\begin{tabular}{|c|c|c|c|}
\hline $\mathrm{M}(\mathrm{TeV})$ & $m_{\chi_{1}^{0}}(\mathrm{GeV})$ & $B\left(\chi_{1}^{0} \rightarrow \gamma \tilde{G}\right)$ & $B\left(\chi_{1}^{0} \rightarrow Z \tilde{G}\right)$ \\
\hline & & & \\
160 & 111.5 & 0.996 & 0.004 \\
190 & 132.0 & 0.975 & 0.025 \\
220 & 152.4 & 0.943 & 0.057 \\
250 & 172.5 & 0.910 & 0.090 \\
280 & 192.3 & 0.878 & 0.122 \\
310 & 211.5 & 0.846 & 0.154 \\
340 & 229.8 & 0.812 & 0.188 \\
370 & 246.4 & 0.767 & 0.233 \\
& & & \\
\hline
\end{tabular}

Table 1

The branching ratios of the lightest neutralino that can be produced in the NLC with $\sqrt{s}=500$ $\mathrm{GeV}$, to a photon and to a $Z$ (alongwith a $\tilde{G}$ in each case). We have chosen $\mu=-300 \mathrm{GeV}$, $\tan \beta=10$ and $M / \Lambda=2$. The decay of the NLSP to a Higgs is negligible. 


\section{References}

[1] For introductory reviews, see, e.g., H.P. Nilles, Phys. Rep. 110 (1984) 1; H. Haber and G.L. Kane, Phys. Rep. 117 (1985) 75.

[2] M. Dine and W. Fischler, Nucl. Phys. B204 (1982) 346; L. Alvarez-Gaumé, M. Claudson and M.B. Wise, Nucl. Phys. B207 (1982) 96; S. Dimopoulos and S. Raby, Nucl. Phys. B219 (1983) 479.

[3] M. Dine and A.E. Nelson, Phys. Rev. D48 (1993) 1277; M. Dine, A.E. Nelson and Y. Shirman, Phys. Rev. D51 (1995) 1362; M. Dine et al, Phys. Rev. D53 (1996) 2658.

[4] S. Dimopoulos and G.F. Giudice, Phys. Lett. B393 (1997) 72; G. Dvali and M. Shifman, Phys. Lett. B399 (1997) 60; M. Hotta, Izawa K.-I. and T. Yanagida, Phys. Rev. D55 (1997) 415; S.P. Martin, Phys. Rev. D55 (1997) 3177; L. Randall, hep-ph/9612426.

[5] J. Bagger et al, Phys. Rev. Lett. 78 (1997) 1002, (E) ibid 78 (1997) 2497; Phys. Rev. D55 (1997) 3188; S. Dimopoulos, S. Thomas and J.D. Wells, Nucl. Phys. B488 (1997) 39.

[6] A. Riotto, O. Törnkvist and R.N. Mohapatra, Phys. Lett. B388 (1996) 599; N. ArkaniHamed, J. March-Russell and H. Murayama, hep-ph/9701286; F. Borzumati, hep$\mathrm{ph} / 9702307$.

[7] R. Barbieri, L. Hall and A. Strumia, Nucl. Phys. B455 (1995) 219.

[8] S. Dimopoulos et al, Phys. Rev. Lett. 76 (1996) 3494; Phys. Rev. D54 (1996) 3283; S. Ambrosanio et al, Phys. Rev. Lett. 76 (1996) 3498; Phys. Rev. D54 (1996) 5395; H. Baer et al, Phys. Rev. D55 (1997) 4463. 
[9] S. Ambrosanio, G. Kribs and S.P. Martin, Phys. Rev. D56 (1997) 1761; J.L. Lopez, D.V. Nanopoulos and A. Zichichi, Phys. Rev. Lett. 77 (1996) 5168; D.A. Dicus, B. Dutta and S. Nandi, Phys. Rev. Lett. 78 (1997) 3055.

[10] A. Ghosal, A. Kundu and B. Mukhopadhyaya, Phys. Rev. D56 (1997) 504.

[11] P. Fayet, Phys. Lett. B70 (1977) 461, ibid B86 (1979) 272, ibid B175 (1986) 471; N. Cabibbo, G.R. Farrar and L. Maiani, Phys. Lett. B105 (1981) 105.

[12] T. Gherghetta, Nucl. Phys. B485 (1997) 25.

[13] K.J.F. Gaemers, R. Gastmans and F.M. Renard, Phys. Rev. D19 (1979) 1605; M. Caffo, R. Gatto and E. Remiddi, Nucl. Phys. B286 (1986) 293; F.A. Berends et al, Nucl. Phys. B301 (1988) 583.

[14] A. Datta, A. Datta and S. Raychaudhuri, hep-ph/9605432 (to appear in Z. Phys. C).

[15] C. Chen, M. Drees and J.F. Gunion, Phys. Rev. Lett. 76 (1996) 2002.

[16] C.Y. Prescott, in Proceedings of the Workshop on Physics and Experiments with Linear $e^{+} e^{-}$Colliders, eds. F.A. Harris et al (World Scientific, Singapore, 1993) p. 379.

[17] See, for example, V. Barger and T. Han, Phys. Lett. B212 (1988) 117.

[18] K. Grassie and P.N. Pandita, Phys. Rev. D30 (1984) 22.

[19] A. Datta, B. Mukhopadhyaya and M. Guchait, Mod. Phys. Lett. A10 (1995) 1011; S. Chakraborty, A. Datta and M. Guchait, Z. Phys. C68 (1995) 325; A. Datta, A. Datta and S. Raychaudhuri, Phys. Lett. B349 (1995) 113; A. Datta, M. Drees and M. Guchait, Z. Phys. C69 (1996) 347; A. Datta, M. Guchait and N. Parua, Phys. Lett. B395 (1997) 54. 


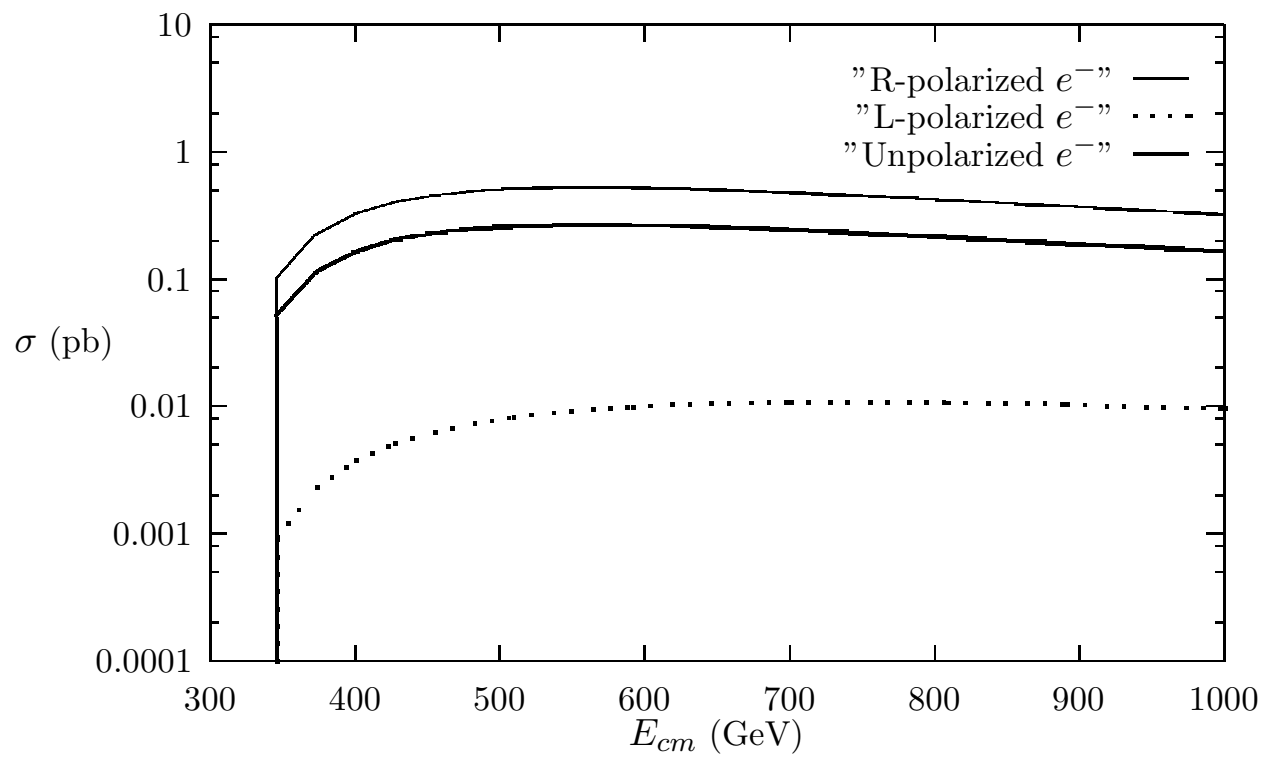

Fig. 1

The cross-section for $e^{+} e^{-} \longrightarrow \chi_{1}^{0} \chi_{1}^{0}$ against the center-of-mass energy, for $\mu=-300 \mathrm{GeV}$, $\tan \beta=10, M=230000 \mathrm{GeV}$ and $M / \Lambda=2$ (which yields $\left.m_{\chi_{1}^{0}}=159 \mathrm{GeV}\right)$. 


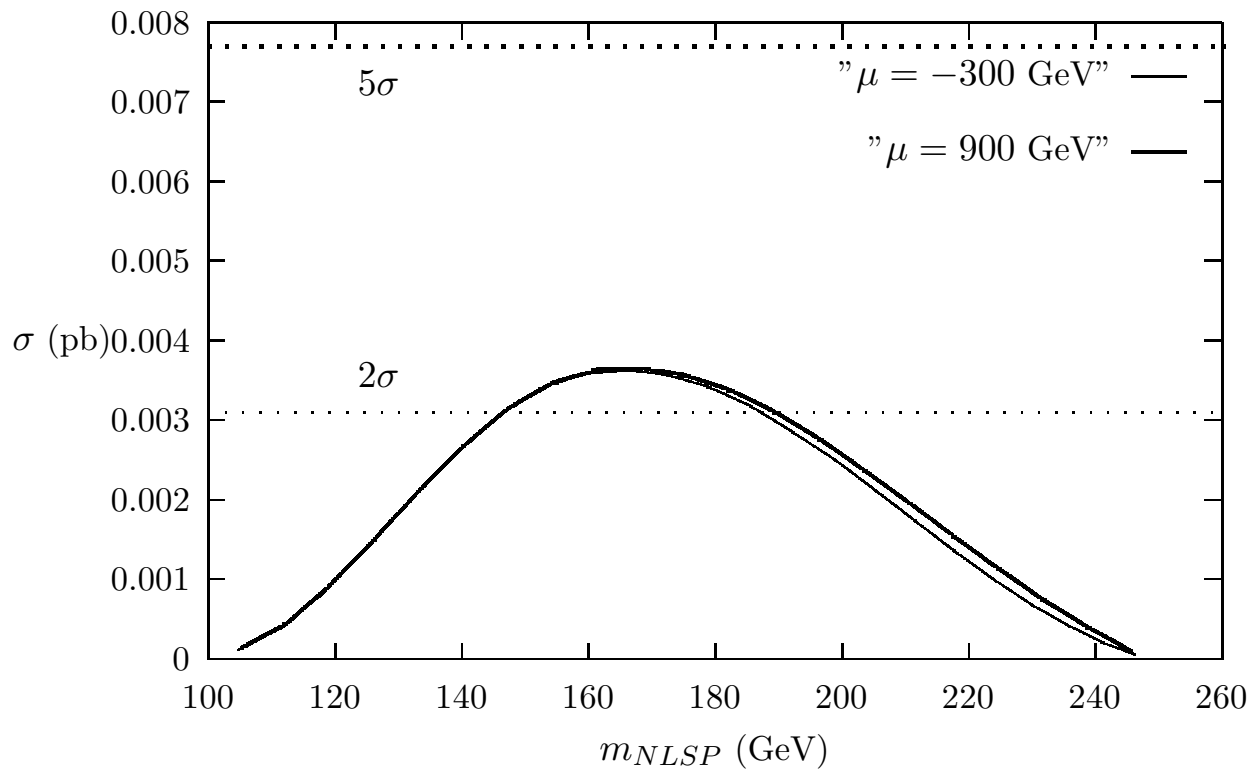

Fig. 2

The cross section for $e^{+} e^{-} \longrightarrow \gamma+$ missing energy against the NLSP mass with unpolarized electron beams at $E_{c m}=500 \mathrm{GeV}$. Two values of $\mu$ are used with $\tan \beta=10$ and $M / \Lambda=2$. Different levels of significance correspond to an integrated luminosity of $30 \mathrm{fb}^{-1}$. 


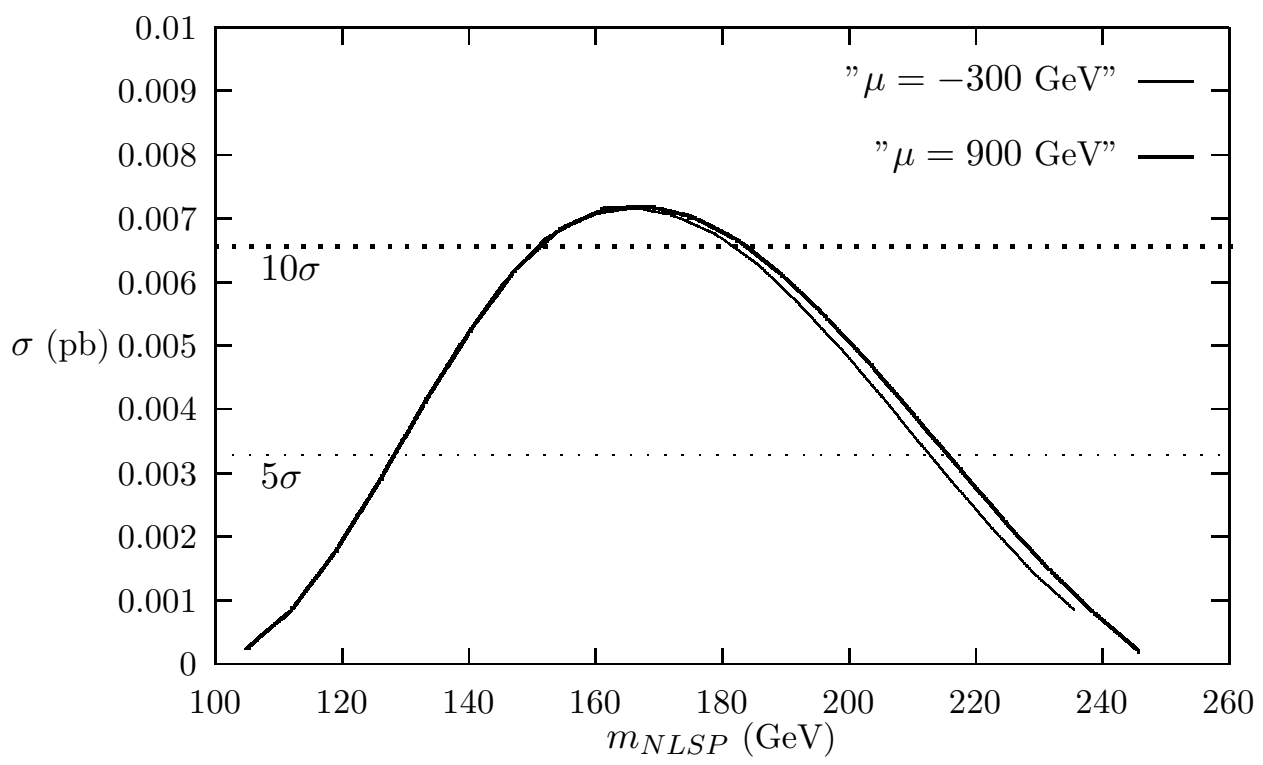

Fig. 3

Same as in Figure 2, but with $100 \%$ right-polarized electron beams. Also, different levels of significance correspond to an integrated luminosity of $10 \mathrm{fb}^{-1}$. 


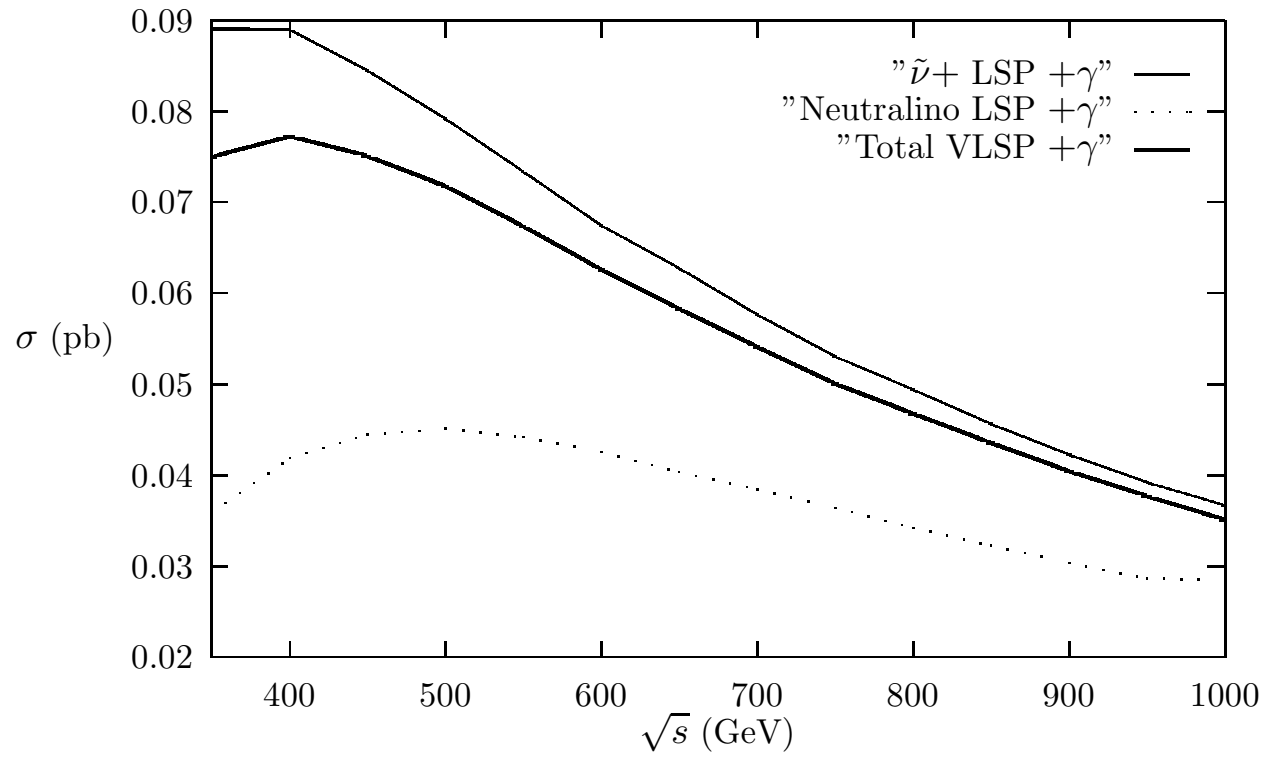

Fig. 4

Single photon production as a function of total center-of-mass energy $\sqrt{s}$. The three lines are drawn with different set of parameters: (i) the contribution with $\tilde{\nu}$ and LSP (lightest neutralino) at $m_{\chi_{1}^{0}}=71 \mathrm{GeV}$ and $m_{\tilde{\nu}}=100 \mathrm{GeV}$; (ii) the contribution with Virtual LSPs $(\tilde{\nu}$ and two lightest neutralinos) at $m_{\chi_{1}^{0}}=71 \mathrm{GeV}$ and $m_{\tilde{\nu}}=120 \mathrm{GeV}$; (iii) the contribution with LSP only at $m_{\chi_{1}^{0}}=100 \mathrm{GeV}$ and $m_{\tilde{\nu}}=150 \mathrm{GeV}$. Gauge coupling unification is assumed. 


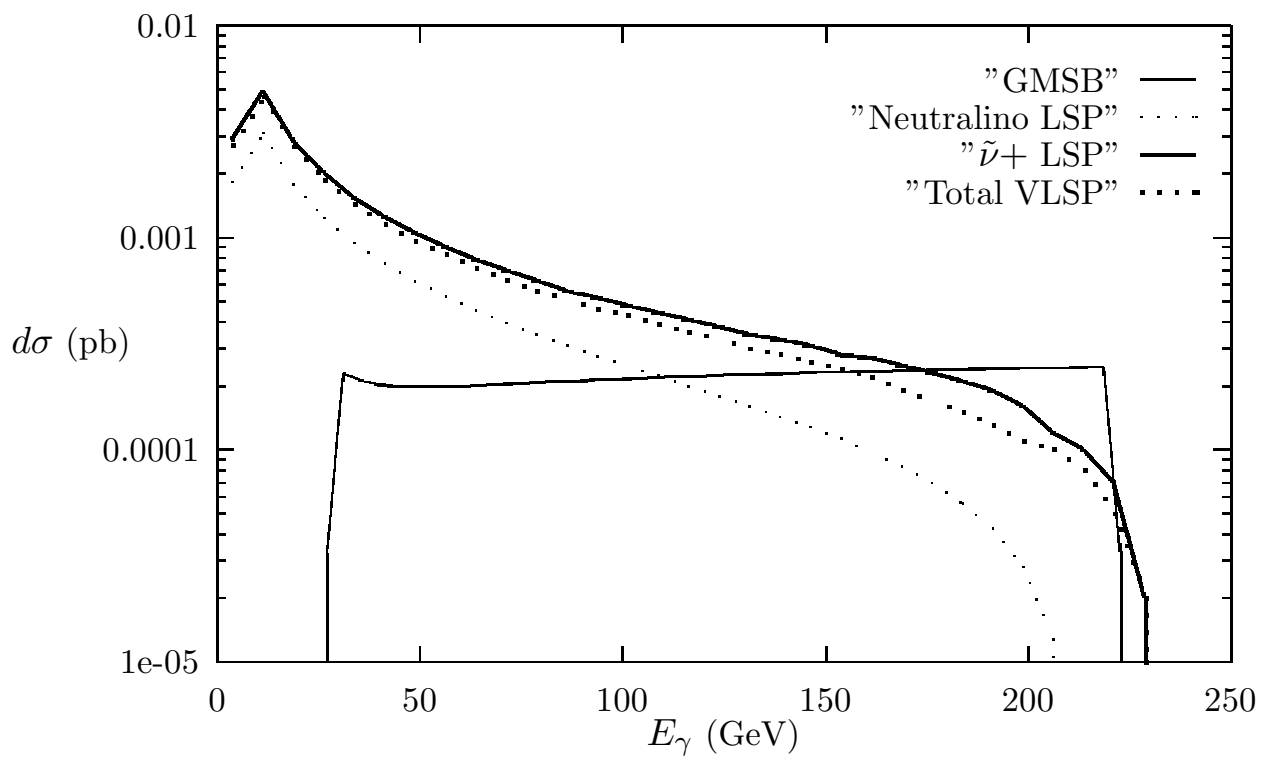

Fig. 5

Energy distribution of the emitted photon with angular cuts as stated in the text. The parameter space for GMSB is the same as in Figure 1, while for MSSM, the three curves correspond to the parameter sets shown in Figure 4. 


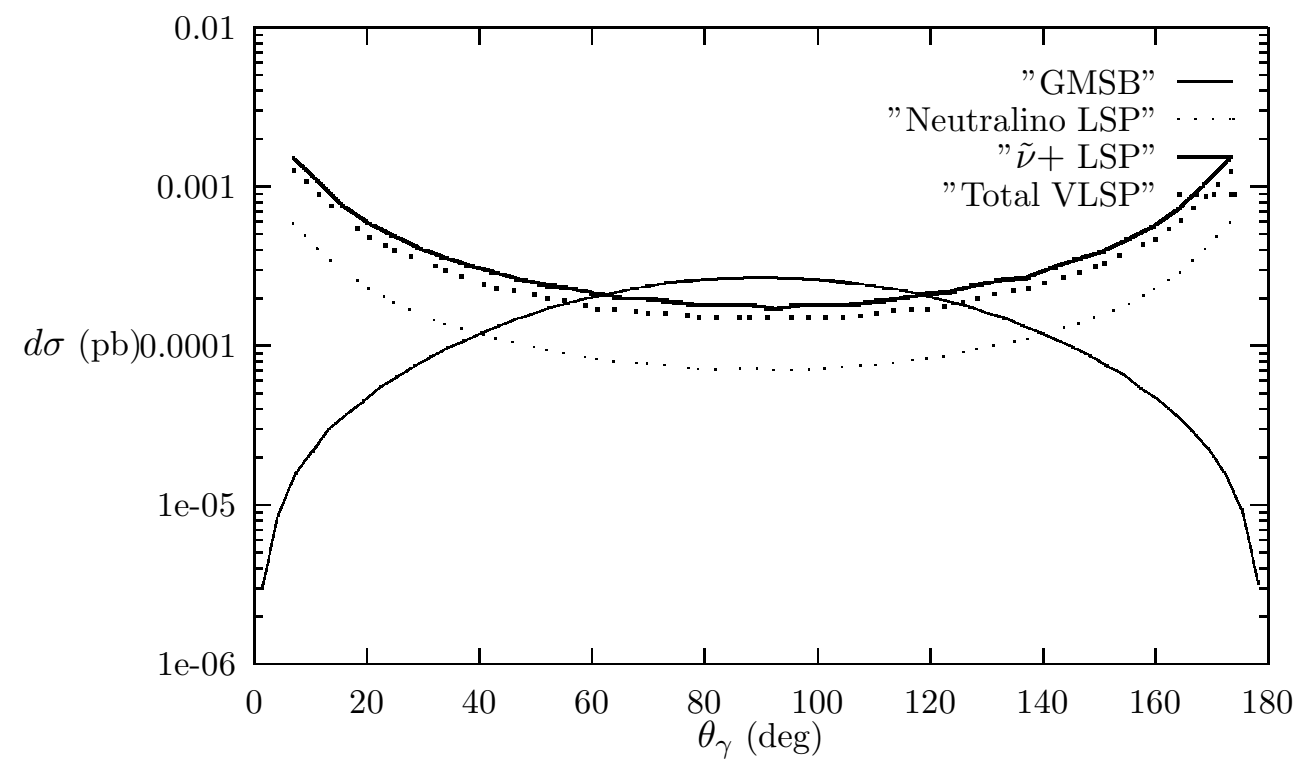

Fig. 6

Angular distribution of the emitted photon with energy cuts as stated in the text. The parameter space for GMSB is the same as in Figure 1, while for MSSM, the three curves correspond to the parameter sets shown in Figure 4. 